\title{
Riesgos laborales en la construcción. Un análisis sociocultural
}

\section{Occupational hazards in construction. A sociocultural analysis}

\author{
Javier Eloy Martínez Guirao ${ }^{1}$ \\ j.eloymartinez@um.es
}

\begin{abstract}
Resumen
A pesar de la medidas que desde hace unos años se adoptan para evitar los riesgos laborales en España, los accidentes y enfermedades producidos por el trabajo siguen ocurriendo y el sector de la construcción es uno de los más afectados. En este artículo, basado en una investigación empírica, pretendemos aproximarnos a las dificultades que se presentan a la hora de adoptar las medidas de prevención. Partimos de la idea de que en los entornos laborales, se genera un conjunto de costumbres, valores e ideas y se retroalimentan con la sociedad en general, dando lugar a las culturas del trabajo. Analizamos, entre otros aspectos, el papel que conceptos como el riesgo, salud, azar, masculinidad, comodidad o incomodidad, tienen a la hora de asumir o rechazar las medidas preventivas, así como en la asunción de riesgos.
\end{abstract}

\section{Palabras clave}

Riesgo, trabajo, construcción, cultura.

\begin{abstract}
Despite the measures in recent years taken in the prevention of occupational risks in Spain, accidents and illnesses caused by work continue to occur and the construction sector is one of the most affected. In this article, based on empirical research, I intend to approach the difficulties that arise when adopting preventive measures. I start from the idea that in work environments are generated a set of customs, values and ideas and feed back to society in general, resulting in cultures of work. Analizing, among other things, the role that ideas such as risk, health, random, masculinity, comfort or discomfort, has in assume or reject preventive measures as well as in risk taking.
\end{abstract}

\section{Keywords}

Risk, work, construction, culture.

Forma sugerida de citar: $\quad$ Martínez Guirao, J.E. (2015). Riesgos laborales en la construcción. Un análisis sociocultural. Universitas, XIII (23), pp. 65-86.

1 Doctor en Antropología social y cultural. Profesor en la Universidad de Murcia, área de Antropología social, departamento de Filosofía, España. 


\section{Introducción}

El trabajo de la construcción, especialmente aquel que se realiza "a pie de obra", es uno de los que presenta una mayor siniestralidad laboral en España. Y pese a que en los últimos años, la prevención de riesgos laborales se ha reconocido como uno de los medios más adecuados para mejorar las condiciones laborales y la calidad de vida en general, y las políticas al respecto, promulgadas por la administración y secundadas por las empresas son cada vez más estrictas, ampliando la concepción del riesgo y prestando atención a factores que en otras épocas pasaban desapercibidos, los accidentes y enfermedades producidos en y por el trabajo siguen ocurriendo.

Muchas veces, la causa es que no se adoptan las medidas necesarias, bien por desconocimiento, por la interferencia de factores económicos, o sobre todo, por la incidencia de la propia cultura.

En efecto, Douglas y Wildavsky (1982) y Douglas (1996) en su teoría cultural del riesgo, nos hablan de cómo la cultura es el principio codificador por el que se reconocen los peligros y condiciona el modo como se perciben los riesgos, y destacan el papel de la interacción social, al estar el sujeto social inserto en entramados de vínculos sociales, de modo que una reputación de temeridad, bajeza, locura o cobardía interferiría en la fortaleza de estos vínculos.

Igualmente, para entender qué sucede debemos incluir la idea de que en los diferentes puestos que se ocupan y en cada una de las empresas, es decir, en el entorno laboral, se generan un conjunto de costumbres, valores e ideas y se retroalimentan con la sociedad en general. Es lo que denominamos las "culturas del trabajo" (Moreno, 1991) que predisponen, además, a percepciones concretas del riesgo.

Con este estudio pretendemos aproximarnos a la realidad de estos hechos, prestando atención a las dificultades que se presentan a la hora de adoptar las medidas de prevención, para encontrar sus causas.

Nos acercamos a factores culturales como la salud, el azar, las creencias y convicciones, las coacciones y obligaciones, el género, la comodidad o incomodidad, las consecuencias derivadas del sistema capitalista basado en la producción, las construcciones y percepciones que se hacen en torno al riesgo y al peligro, etc.

El análisis de estos factores nos ayudará a comprender la propia noción riesgo como un constructo cultural, que guía las actuaciones preventivas de los sujetos sociales. 


\section{Metodología ${ }^{2}$}

La investigación se ha realizado en seis obras de construcción que se estaban desarrollando en la Comunidad Valenciana (España). Cuatro de ellas eran edificios de viviendas de varias plantas, tres en la provincia de Alicante y una en la de Valencia, mientras que las otras dos consistían en la edificación de viviendas unifamiliares, ubicadas en la provincia alicantina.

Para ello, realizamos un trabajo de campo de unos cuatro meses de duración, acudiendo con frecuencia a las respectivas obras para recoger información. Nuestra intención ha sido realizar una investigación aproximativa desde un punto de vista antropológico, que incite a posteriores estudios con mayor profundidad.

Hemos partido de la idea de que no es lo mismo lo que la gente dice, que lo que la gente hace, o lo que la gente dice que hace; o incluso, lo que la gente piensa que hace, que lo que hace realmente. Conseguir captar todos estos aspectos de la realidad a estudiar solo se hace posible a través de una combinación de técnicas en el trabajo de campo etnográfico.

Por ello, en esta investigación hemos aplicado tanto técnicas cuantitativas como cualitativas. Concretamente, las herramientas utilizadas para la recogida de información han sido: la observación y la entrevista abierta como técnicas cualitativas; la encuesta, como técnica cuantitativa; y a medio camino entre estos dos tipos de técnicas, la entrevista estructurada. Su utilización está fundamentada en la triangulación, ya que separadamente presentan limitaciones que mediante la combinación de todas pueden ser subsanables. Así se puede obtener información en forma de datos cuantificables que al conjugarse con la información cualitativa permite que se establezcan relaciones entre ambas que nos ayudan a reorientar la investigación hacia datos más relevantes.

La observación nos ha proporcionado la posibilidad de ver in situ, pudiendo explorar las relaciones entre "lo que se dice", "lo que se dice que se hace", "lo que se debería hacer" y "lo que en realidad se hace", dentro del propio contexto en el que se producen los acontecimientos. A su vez nos ha ofrecido un adecuado conocimiento microsocial del tema que presentamos.

2 Este artículo está basado y hace uso de los datos recogidos a través de dos proyectos de investigación ( $L a$ percepción del riesgo en el sector de la construcción y La percepción del riesgo en empresas y trabajadores de la Comunidad Valenciana), encargados por Ateco y Nodos Habitat. El equipo que ha recogido los datos de campo estaba formado, además del autor del texto, por Maite Moya, Beatriz Serrano y Teresa Antón. Junto a ellos se ha utilizado otra información recopilada exclusivamente por el autor para la elaboración del artículo. 
Las entrevistas abiertas han ido enfocadas a obtener información en profundidad que nos ha permitido comprender la perspectiva emic ${ }^{3}$ de la realidad, es decir, la significación de los discursos de los informantes. Esto nos ha facilitado conocer los porqués de las relaciones entre los factores culturales, la percepción y la actuación de los trabajadores en relación al riesgo y desde una perspectiva de género.

La entrevista estructurada se ha orientado a obtener información sistemática en relación con las categorías de análisis y observación, ampliando así los datos.

La información más cualitativa obtenida a través de las entrevistas se ha complementado con las encuestas, en un total de 102, que nos han posibilitado recoger datos cuantitativos y añadir, en cierto modo, el factor de representatividad al de profundidad. Si bien este último aspecto es el que hemos pretendido que sea más relevante.

Los informantes han sido los propios albañiles, en sus diversas categorías profesionales, técnicos y delegados de prevención y empresarios.

\section{El trabajo de la construcción}

El sector de la construcción requiere de una variedad considerable de tareas con diferentes características y situaciones concretas de riesgo.

Las obras constan de varias fases sucesivas, con elevados índices de siniestralidad, como los movimientos de tierra, la cimentación, la construcción de las estructuras (que incluyen encofrados, trabajos con ferralla y con hormigón), los cerramientos, las cubiertas, los acabados y las instalaciones (fontanería, calefacción, electricidad, etc.).

Aunque se hace difícil precisar actividades que, "a pie de obra", entrañen más riesgos que otras, muchos de nuestros informantes colocaban el trabajo en el andamiaje exterior como uno de los más arriesgados, donde algunos afirmaban, además, que a veces no se cumplían las normativas para la prevención de riesgos laborales. Igualmente, también se hace alusión con relativa frecuencia, por ejemplo, a la cimentación y al encofrado de la obra, o en la especialidad de electricista al montaje y puesta en funcionamiento de los contadores de luz, por el riesgo de electrocución y de sufrir quemaduras.

3 Cuando hablamos del punto de vista emic nos referimos a la propia perspectiva del sujeto social. 
En este tipo de trabajos se está expuesto a múltiples situaciones de riesgo, siendo las más frecuentes las derivadas de posturas incómodas, mover cargas pesadas, caídas desde diferentes alturas, caídas de objetos o herramientas, golpes, cortes, quemaduras, etc.

Para minimizar los riesgos y evitar que estos se materialicen en accidentes o enfermedades, la legislación obliga tener una serie de planes de actuación y protocolos preventivos y realiza inspecciones periódicas para supervisar su implantación.

De este modo, las empresas se ven obligadas a acatar acciones en beneficio de la seguridad de los trabajadores, como es el proporcionar y obligar a usar los equipos de protección individual (EPI's) o diversos medios de prevención colectivos, así como el formar e informar de los riesgos y de las mejores maneras para evitarlos (BOE, 2012, 2015).

\section{La formación-información como medida de prevención}

Son numerosos los autores que afirman que la información transmitida y la formación recibida no garantizan la percepción de los riesgos por parte de la población (Beck, 1986; Douglas, 1996; Slovic, Fichtenstein y Fischhoff, 1979) y que posiblemente la educación no sea la medida de actuación más adecuada. Como señala Bestard (1996, p. 15), haciendo alusión a Douglas, esta concepción implica entender que el problema de la percepción de los riesgos es más complejo que la correcta aprehensión por parte de la sociedad del discurso experto, y las decisiones racionales sobre la exposición a determinados peligros en función de los posibles beneficios. Así, para Douglas "la percepción también depende de las nociones de justicia presentes en una sociedad e involucra dimensiones éticas, morales y de credibilidad institucional que pueden variar de sociedad en sociedad y entre los grupos de una misma unidad social" (Montenegro, 2005, p. 124).

Investigaciones como la de Borgia et al. (1997) sobre un grupo de agricultores italianos parecen reafirmar esta idea. Los autores concluyeron que los cursos de formación que habían recibido los trabajadores sobre el manejo de plaguicidas no eran garantía de su uso correcto, debido, principalmente, a la poca receptividad que había por parte los agricultores (Espluga, 2004, p. 36).

No obstante, la mayoría de las intervenciones que se hacen desde los poderes públicos apuestan por una mejora de la comprensión de la información, así como 
de campañas informativas que produzcan una mayor sensibilización (Frías, 2006, p. 36). Esta estrategia aparece en todo el corpus legal sobre la prevención de riesgos laborales, donde la formación e información son ejes básicos ${ }^{4}$.

\section{Para autores como Cobos, Pérez y Reyes:}

La formación en la empresa no es la solución a todos los retos de la prevención de riesgos pero sí una técnica útil y necesaria, seguramente fundamental, para desarrollar el clima preventivo (...) para interiorizar estos aspectos es necesaria una predisposición previa y es aquí donde la formación entronca con el concepto de cultura preventiva. Desarrollar esta predisposición hacia una cultura de seguridad, de la prevención, supone trabajar sobre valores, actitudes y comportamientos del conjunto de la sociedad y de sus colectivos y organizaciones para avanzar de manera estable y permanente en la mejora de las condiciones de trabajo (2011, p. 86).

Por ello, vamos exponer la cantidad y calidad de formación e información que los trabajadores afirman tener, partiendo de datos cuantitativos que iremos matizando.

En principio, el $72 \%^{5}$ de los encuestados afirma conocer la Ley de Prevención de Riesgos Laborales, mientras que un 27\% dice lo contrario.

Con respecto a la formación en prevención de riesgos laborales, el 58\% asegura haber realizado algún curso, mientras que un significativo $35 \%$ menciona que no tiene ningún tipo de formación al respecto. Este porcentaje, por supuesto, varía de unas empresas a otras. En algunas de ellas, los delegados de prevención de riesgos laborales tratan de mantener una formación continua en los trabajadores, para la que generalmente se imparten charlas anuales a través del servicio de prevención externo.

De ese $58 \%$ que dice tener formación, tan sólo un $64 \%$ considera la información recibida como útil, mientras que el $35 \%$ no está muy conforme con la formación que se les ha dado, por considerarla poco centrada en sus necesida-

4 La Directiva Marco Europea (Unión Europea, 1989) y las directivas derivadas de ella, la Estrategia Europea para la Seguridad y Salud en el Trabajo (2007-2012), el Estatuto de los Trabajadores (España, 1995), la Ley de Prevención de Riesgos Laborales (España, 1995, 2003) y todos los Reales Decretos que la desarrollan, la Estrategia Española de Seguridad y Salud en el Trabajo (2007-2012) (Cobos, Pérez y Reyes, 2011: p. 76), así como el V Convenio Colectivo del Sector de la Construcción (España, 2012) dan especial importancia a la formación e información.

5 Muchas de las preguntas realizadas constaban de múltiples respuestas. En este artículo vamos a utilizar en las interpretaciones solamente los datos más relevantes, sin entrar a valorar las respuestas con bajo porcentaje. Es por ello por lo que la suma entre los porcentajes referidos a la misma cuestión no siempre alcanzarán la cifra del 100\%. 
des. Y es que según los datos obtenidos de la encuestas y en las entrevistas, ya sea por los contenidos en sí mismos o por las metodologías pedagógicas que se utilizan, parece haber una distancia importante entre las necesidades de los trabajadores y las medidas aportadas, que se acentúa por su escasa participación en las medidas de prevención que se implantan. Así, menos de la mitad de los encuestados, el $49 \%$ afirman que son consultados por la empresa y que participan en materia preventiva. En este sentido una actitud más dialógica podría permitir construir los planes de prevención atendiendo a las necesidades tanto de las empresas como de los trabajadores.

Otro aspecto está relacionado con la información que los trabajadores reciben, que pudiere no ser suficiente, según los datos recogidos. Sólo el $67 \%$ menciona que la empresa le ha informado de los riesgos específicos de su puesto de trabajo antes de iniciar el trabajo. Un 21\% habría recibido la información un tiempo después de haber iniciado la actividad, a un 10\% no le habría informado la empresa, sino otros compañeros, y un $2 \%$ nunca habrían recibido información al respecto.

Por otro lado, el 98\% considera necesario recibir formación en prevención de riesgos laborales. No obstante, en la formación dentro de las empresas se percibe un problema relacionado con la idea de que el tiempo que se dedica a ella puede entenderse como no productivo ${ }^{6}$. De modo, que si en las encuestas nos dicen que, tanto empresarios como técnicos y trabajadores, consideran casi de manera unánime la necesidad de formación en prevención de riesgos, es en las entrevistas abiertas y en los diálogos más informales cuando hemos podido apreciar que hay otros factores que inciden, además de lo políticamente correcto, en los discursos de los informantes y probablemente también en sus prácticas.

\section{EI uso de las medidas de protección individuales y colectivas}

Como suele suceder, pese al anonimato de las encuestas, las respuestas, más que lo que ocurre en la realidad, lo que nos indican son los valores compartidos, los considerados como deseables. De este modo, según nuestra interpretación, tras la triangulación con otras técnicas, vemos que el 95\% de los tra-

6 Según autores como Reig y Clemente (2010) la pérdida de competitividad y rentabilidad por el tiempo dedicado a la formación en prevención de riesgos laborales es una creencia generalizada por parte de empresarios y directivos. 
bajadores son conscientes de la necesidad del uso de los EPI's, y de que cuando no las usan, están haciendo algo mal.

Otro dato, que habla de la buena aceptación que parecen tener las medidas de protección es que estas son suficientes para un $90 \%$.

El porcentaje crítico hacia ellas aumenta hasta el 12\% cuando incluimos, junto a los EPI's los medios de protección colectivos (barandillas, vallas, etc.) y aludimos a si entorpecen o mejoran el trabajo. Un 70\% dice lo mejoran, pero, en este caso, al igual que en anterior, los porcentajes no son más que meros indicadores de valores y del conocimiento de la legislación que impone el uso de las medidas como obligación, con sus consecuentes sanciones a quien la transgreda. La metodología cualitativa utilizada nos ha permitido entender cómo para un importante número de empresarios y trabajadores, las medidas de protección entorpecen su trabajo y les hacen perder un tiempo considerable, a la vez que les resta habilidades manuales, lo que provoca, junto a otros factores, que muchos de ellos no las usen en todas las situaciones.

Por ejemplo, un técnico nos contaba cómo tienen muchas dificultades para que los empleados hagan uso del casco, otros hacen alusión a los guantes, las mascarillas, las gafas o los arneses.

De hecho, la contradicción antes señalada, comienza a vislumbrarse, cuando, a pesar de haber afirmado que prácticamente todos usan los EPI's, las cuales consideran necesarias y suficientes, que no entorpecen su trabajo y que se las proporciona sin falta la empresa (según afirma un 98\%), paradójicamente, dicen asumir riesgos en el desempeño de su trabajo un $57 \%$ de los encuestados, porcentaje que aumenta a más de un $66 \%$, cuando hablan de sus compañeros. Evidentemente parece ser más fácil decir que los demás asumen riesgos, a que lo hace uno mismo, sobre todo cuando puede ser algo que esté mal visto; o decir que se toman riesgos, con la ambigüedad que el enunciado denota, que decir que no se usan los EPI's, lo cual supondría reconocer el no cumplir con una obligación?

El exceso de confianza es uno de los factores principales, desde el punto de vista emic de los propios informantes, que provoca que en muchas ocasiones los

7 Los resultados contrastan, en cierto modo, con los obtenidos en otras investigaciones realizadas en España en otros sectores laborales, como la que desarrolló Espluga (2004) sobre los agricultores catalanes, debido a que se trataba de "trabajadores autónomos" y que, por tanto, durante el transcurso de la investigación no estaban sujetos a la Ley de Prevención de Riesgos Laborales (LPTR 31/1995), por lo cual no tenían obligación legal de cumplir las normas de prevención y seguridad más allá de aquellas que pudieren provocar daños a terceros. En su estudio los agricultores reconocen no hacer uso frecuentemente de los EPI's cuando trabajan con plaguicidas, y cuando lo hacen es con muchas deficiencias y poca regularidad. 
trabajadores no sean conscientes de los peligros que implica su actividad cuando no se ejecuta correctamente y dejen de usar los EPI's. Algunos empresarios se quejaban de que los trabajadores provocan problemas a las empresas en materia de prevención laboral, donde, según afirmaban, la legislación las perjudica.

\section{Las culturas del trabajo}

En cada tipo de trabajo, en cada empresa concreta, y en cada puesto laboral se generan unos hábitos, unas costumbres, unos comportamientos, unos valores que se transmiten desde los jefes, delegados de prevención y trabajadores más experimentados a los más nóveles, y que tienden a perpetuarse en el tiempo, aunque con cambios más o menos significativos, según las actuaciones al respecto que se vayan tomando. Ese conjunto de comportamientos y valores es lo que denominamos cultura del trabajo, que a su vez puede conllevar un mayor o menor grado de prevención. Como afirma Moreno:

El proceso de trabajo en que cada individuo está inmerso (...) y la posición que se ocupa en este proceso, la cual viene determinada por la división social del trabajo que producen las relaciones sociales de producción en que dicho proceso tiene lugar, se hallan en la base no sólo de las condiciones materiales de existencia sino que condicionan e impregnan todos los ámbitos de la vida: desde las opciones o estrategias matrimoniales y el tipo de relaciones intrafamiliares o de vecindad, hasta la forma de representarse el mundo y de expresar los sentimientos. Se genera no sólo una cultura sobre el trabajo, sobre todos los diversos aspectos de la esfera laboral, sino también una cultura desde el trabajo, a partir de los diversos procesos de trabajo y lugares ocupados en las relaciones sociales de producción: por eso es preciso hablar, no en singular sino en plural, de culturas del trabajo (1991, p. 619).

De este modo dan lugar a las denominadas culturas del trabajo, "entendiendo por tales las que se generan en los diversos procesos de trabajo desde la ocupación de distintas posiciones en las relaciones de producción" (Moreno, 1991, p. 617). Estas, a su vez, producirían diferentes identidades socioprofesionales, definiéndolas como aquellas que diferencian como colectivos a los grupos que las comparten y que se construyen "sobre la base de la participación de los sujetos en un determinado proceso de trabajo y desde una misma posición en las relaciones sociales de producción” (Téllez, 2002, p. 194). 
En este sentido, siguiendo a Urteaga (2012, pp. 50-51), en ocasiones, la asunción de riesgos puede convertirse en un recurso identitario. Así lo ilustra el estudio que Zonabend (1989) sobre unos obreros en una fábrica de La Hague, expuestos laboralmente a contaminación radiactiva:

Durante los cursillos de formación, la dirección de la entidad intenta desdramatizar ese riesgo. Para subrayar hasta qué punto el trabajo solicitado es simple e inofensivo, los formadores lo comparan con las actividades domésticas ordinarias: limpiar, lavar la ropa, cocinar, etc. Pero, estas comparaciones no son del gusto de los obreros, que desearían construir una identidad profesional más viril: desean ser verdaderos hombres y ser los "guerreros de la energía nuclear", en lugar de ser las "amas de casa del átomo": su modelo profesional es el minero del siglo XIX, que arriesga cada día su vida exponiéndose a los grisú y a los desprendimientos. Jugar con el riesgo de contaminación les permite afirmar esta identidad: la primera irradiación aparece entonces como un rito para convertirse en un verdadero obrero (Urteaga, 2012, pp. 50-51).

No obstante, hemos de precisar que los ámbitos laborales forman una parte de las vidas y el tiempo de los trabajadores, quienes desarrollan sus cotidianidades en diferentes contextos que implican a su vez diversas socializaciones secundarias.

Así, las culturas del trabajo ${ }^{8}$, siempre estarán influenciadas y condicionadas por otros niveles culturales más macrosociales y por las políticas y legislaciones respecto a la prevención de riesgos laborales. Por ello, como hemos señalado, pensamos que estas culturas son susceptibles, en cierta medida, de cambio a través de leyes y campañas de concienciación.

\section{Riesgo y peligro como constructos sociales}

Un aspecto fundamental que hemos tenido en cuenta en nuestra investigación es que para el uso de las medidas de protección, el trabajador debe ser consciente del riesgo que corre en el desempeño de su trabajo, y este es un hecho que no siempre resulta tan evidente.

8 El estudio de las culturas del trabajo se complejiza cuando añadimos factores como la edad, el género o la etnia. A modo de ejemplo hemos encontrado diferencias significativas en los comportamientos entre trabajadores procedentes de Rumanía, Marruecos, algunos países de Sudamérica y España. Un análisis más profundo de todos ellos está previsto para futuras investigaciones, mientras que en ésta nos centramos principalmente en los trabajadores oriundos de la zona geográfica estudiada. 
Son diferentes los factores que atañen a cómo es entendido el riesgo, que parten de la información y formación que los trabajadores reciben, pero que, en muchas ocasiones, la trascienden. Algunos son producidos directamente en las culturas propias de los trabajos y empresas donde desempeñan su actividad. Otros van más allá y están basados en los valores considerados hegemónicos por la sociedad en general y la cultura.

De hecho, como ya adelantara Beck (1986), el riesgo constituye una nueva forma de división social con relación al conocimiento. No todo el mundo es consciente de los riesgos y peligros que se dan en sus situaciones cotidianas y laborales. De ahí la importancia de la formación y los cursos de prevención. Pero también sabemos que, aun conociendo esas posibles situaciones de riesgo, algunos sujetos, empujados por valores propios de la juventud o de la masculinidad, o incluso la misma concepción del azar, que hemos encontrado entre muchos de los trabajadores entrevistados, pueden llegar a sentir cierta atracción por situaciones de riesgo.

Por otro lado, en cada tipo de empresa y, muchas veces, en cada empresa concreta, se perciben o se construyen situaciones de riesgo específicas, lo que produce que, en ocasiones, haya discrepancias entre las evaluaciones técnicas del servicio de prevención y opiniones de los miembros de la empresa. Y lo hace en función de las ideas de los empresarios, técnicos y trabajadores y del historial de accidentes en la misma empresa, así como de las propias biografías de los trabajadores.

No obstante, queda patente cierta eficacia de los cursos de prevención de riesgos laborales, así como las actuaciones legislativas a la hora de la concienciación de los trabajadores, hasta el punto de que estos no se planteen siquiera que existan posibles medidas de prevención que no estén contempladas en ellos.

Así, por ejemplo tenemos el caso de la hidratación, que resulta necesaria especialmente en épocas estivales, donde las altas temperaturas han provocado en algunos casos en nuestro país una considerable siniestralidad laboral. Una respuesta común a la clasificación de medidas de prevención según su importancia, que solicitábamos a nuestros informantes, era que el hecho de beber agua no era una medida, tomándose a cierta broma la pregunta.

Lo mismo ocurre con el uso de protecciones de la zona lumbar de la espalda para minimizar el impacto en los discos intervertebrales a la hora de levantar cargas de peso relativamente elevado, lo que suele ser habitual en este tipo de trabajos.

Otro caso significativo es el no uso de cremas de protección solar en verano, a pesar de que los discursos médicos tan difundidos en los medios de comu- 
nicación advierten enérgicamente del peligro que supone la exposición prolongada al sol en nuestras latitudes.

Pero este hecho nos sugiere otra interpretación: los trabajadores consideran peligroso aquello más evidente, más inmediato, inminente o perceptible a corto plazo (Fischhoff et al., 1978). Ese es uno de los motivos por el que elementos como la exposición prolongada al sol no plantea peligro.

En cierto modo habría que achacarlo también a la confusión existente por la recepción de mensajes que trasmiten los medios de comunicación, en algún grado contradictorios en relación con el riesgo. Esta es una característica que, advertía Beck (1986), presenta la sociedad del riesgo actual. Por un lado, se nos transmite la idea de que estamos rodeados de peligros, y por otro se desmienten esos mensajes. Se nos dice que los alimentos transgénicos son peligrosos, el uso de los teléfonos móviles, las antenas de telefonía, los ordenadores, la gripe A, las propias vacunas de la gripe, el ébola, el cambio climático, etc.

Unos mensajes hegemónicos y otros contramensajes que aluden a una conspiración por parte de ciertos lobbies empresariales, de los gobiernos, de las farmacéuticas que en función de beneficios económicos nos ocultan la información. Igualmente se difunden hechos que confirman esta hipótesis, como la exportación de carne contaminada de vacas locas al tercer mundo, la ocultación de ciertos accidentes en centrales nucleares, el accidente de Chernóbil, etc.

Estos factores convergen unos con otros para que aquello que no es evidente, inmediato, tienda a mirarse bajo la sospecha de la exageración.

Otro aspecto que confluye es la descontextualización de las medidas de protección, también adoptadas de las campañas de los medios de comunicación. De modo que, por ejemplo, se piense que el sol es un peligro en la playa, pero no en la obra.

$\mathrm{Y}$ son este tipo de medidas las que pueden tornarse como exageradas $\mathrm{y}$, como veremos en un próximo apartado, en las que un modelo tradicional hegemónico de masculinidad se hace más patente.

La confusión se incrementa al no poder cuantificarse el peligro de ciertos factores. Un trabajador percibe, sabe, que si le cae un ladrillo en la cabeza desde una primera planta le causará lesiones graves. Sin embargo no se cuantifica la cantidad de rayos de sol que deben "caer" sobre la piel para que resulte perjudicial, más allá de leves quemaduras. Las quemaduras del sol pueden asemejarse a los rasguños o magulladuras en las manos, en los brazos o en las piernas, que se observan en numerosos trabajadores, o al aspecto calloso de sus manos.

Siguiendo estos mismos argumentos, el uso de las mascarillas que minimiza la inhalación de agentes tóxicos, polvo de cemento, de ladrillo, etc., es el 
percibido como menos necesario, y por tanto se usan en menor medida (sólo en casos en los que la supervisión de empresa o la evidencia e inmediatez de los daños las hace imprescindibles).

Teniendo en cuenta todos los aspectos señalados, si hablamos porcentualmente, los encuestados sitúan cuatro factores principales que configuran el riesgo percibido y construido en sus lugares de trabajo.

El primero, al que aluden un $25 \%$ de ellos es el que se debe a los esfuerzos o posturas forzadas que, como es evidente, puede provocar lesiones o patologías musculo-esqueléticas de diferente gravedad, como pueden ser, lumbalgias, tendinitis, tirones musculares, esguinces, etc.

El segundo factor, mencionado por un $17 \%$, es el exceso de confianza o costumbre y está relacionado con la incorporación de hábitos incorrectos desde el punto de vista de la prevención de riesgos. Lo cual sugiere a su vez una inclinación hacia factores de probabilidad y azar ("habría que tener muy mala suerte para que sucediera algo en una actividad que resulta tan sencilla"), así como una insuficiente supervisión y formación continua que permita tener presente de manera constante los riesgos y sus implicaciones.

Los factores tercero y cuarto están, ambos, vinculados a la necesidad de mantener una alta productividad por parte de las empresas. Así, el 13\% de los trabajadores hacen referencia al cansancio o la fatiga, mientras que el 13\% lo hacen al acelerado ritmo de trabajo. Entre ambos suman el 26\%, constituyendo el índice más alto de los señalados.

\section{La idea de salud}

El uso de las medidas de prevención puede sustentarse ideológicamente en la necesidad de preservar la salud. El concepto de salud ha ido variando con los años en nuestra cultura simultáneamente a la valorización y culto al cuerpo actual.

$\mathrm{Si}$ en otras épocas la conservación de nuestro cuerpo y, por tanto, de la salud corporal no resultaba demasiado importante, en la actualidad, se ha llegado a sacralizar el cuerpo en tal grado, que han surgido incluso enfermedades relacionadas con comportamiento obsesivos respecto a lo que es sano o no para el cuerpo, como es el caso de la ortorexia.

No obstante, en la obra no encontramos que se de tanta importancia a la salud como en otros ámbitos, y vemos una contraposición clara a la masculinidad hegemónica tradicional machista. Es decir, en un trabajo considerado tan masculino, la excesiva atención a la salud puede considerarse como femenina. 
De este modo, en las encuestas y entrevistas realizadas hemos obtenido los siguientes resultados:

Destaca el alto porcentaje de encuestados que practican deporte habitualmente, el $81 \%$, frente al $9 \%$ que afirman cuidar su alimentación. Es más, el $84 \%$ dicen creer que su alimentación no es del todo sana, o, al menos, que podría serlo más.

La posible contradicción entre los datos correspondientes al deporte y a la dieta, se aclara al indagar sobre el tipo de deportes que realizan.

Como ya hemos señalado en otra ocasión (Martínez Guirao, 2004), el deporte y el ejercicio en la actualidad están relacionados con la salud, la estética y el ocio, adquiriendo cada uno de estos conceptos mayor o menor relevancia en función del contexto.

Las personas que trabajan "a pie de obra" suelen tener por término medio una edad relativamente joven, sobre todo, si tenemos en cuenta el desgaste físico al que sus cuerpos se ven sometidos en el desempeño de sus actividades laborales. Y a estas edades, la salud es un aspecto secundario que queda relegado por la estética y el ocio.

Pero el culto al cuerpo "juvenil" que enfoca su énfasis principalmente en la estética corporal y que requiere de un trabajo casi diario en los gimnasios, se ve matizado cuando hablamos de los trabajadores de la construcción. Así, el deporte practicado por excelencia en estos contextos es el fútbol sala, que se realiza principalmente en fines de semana, seguido muy de lejos por salir a correr. Es evidente que la motivación que impulsa a su práctica es la del ocio.

La falta de tiempo por los amplios horarios de trabajo o el cansancio físico acumulado en las tareas manuales, que cumplirían en parte la función del gimnasio, son factores explicativos de estos resultados.

No obstante, aunque de manera ponderada, no podemos desestimar la influencia que la idea de preservar la salud tiene en la mayor o menor asunción de riesgos.

La variabilidad de los problemas de salud derivados de las actividades laborales es elevada, si bien, tres de ellos se ubican sobre los demás y se refieren a afecciones musculo-esqueléticas: Un 95\% de los entrevistados afirma haber sufrido dolores de espalda como consecuencia de su trabajo, un $42 \%$ menciona dolores en las rodillas y un $41 \%$ problemas en las articulaciones en general. Tan sólo un $10 \%$ habla de heridas.

Pero, al igual que en los conceptos de riesgo-peligro y accidente, también la idea de salud se construye de manera diferente en cada trabajo según la cultura. Lo que, por ejemplo, para un pianista, podía ser un problema de salud, que además le impidiera o al menos le entorpeciera considerablemente su actividad, en 
otros trabajos manuales, aunque también entorpezca y tenga consecuencias laborales, puede minimizarse la percepción que se tiene sobre sus efectos en la salud.

De hecho las contusiones y pequeños hematomas producidos por golpes, los rasguños, arañazos o pequeñas heridas, como pudimos observar eran más frecuentes de lo que aludían, en las manos de los trabajadores o en otras partes del cuerpo. Esto denota tanto que en algunas ocasiones no usan las medidas de protección, como que se trata de lesiones no apreciadas como tales por los trabajadores.

\section{Género, representaciones sobre masculinidad $y$ asunción de riesgos}

\section{Como advierte Téllez (2013):}

El género, igual que el sexo social, es una construcción cultural, pero éste, en nuestra cultura, se denomina género femenino o género masculino. Todo lo que socialmente se considere que es propio de mujeres será catalogado como "femenino" y todo lo que se considere propio de hombres será "masculino". Así, podríamos hablar de trabajos femeninos y masculinos, coches más femeninos o más masculinos, deportes femeninos y masculinos, etc. Ciertamente, tendemos a generizar dicotómicamente gran parte de nuestra realidad, otorgándoles unas supuestas características masculinas y femeninas a actitudes, olores, profesiones, colores, instrumentos musicales, aficiones, sentimientos, etc. (2013, pp. 58-59).

Desde hace unos años se han ido realizando estudios sobre las masculinidades y sus repercusiones. Como afirma Jociles (2001) estos estudios nos han ido mostrando cómo, las concepciones y las prácticas sociales sobre la masculinidad, varían según los tiempos y los lugares, y que "no hay un modelo universal y permanente de la masculinidad, válido para cualquier espacio o cualquier momento".

Estamos de acuerdo con Connell (1995 citado por Minello, 2002), cuando afirma que la masculinidad es una construcción social, por lo que necesariamente cambia de una cultura a otra, incluso dentro de cada cultura en distintos momentos históricos, a lo largo del curso de vida de cada individuo y entre diferentes grupos de hombres en función de su etnia o clase social.

Actualmente, en nuestra sociedad, es posible apreciar una diferente concepción entre masculinidad y feminidad y darnos cuenta de que "es muy raro que se dude de la feminidad de una mujer mientras que la masculinidad de un hombre está siempre 'bajo sospecha"” (Jociles, 2001). 
Esta idea de la masculinidad bajo sospecha puede explicar el porqué de algunos comportamientos que parecen arriesgados por parte de los trabajadores, pues el ámbito de trabajo en la construcción es primordialmente masculino.

De hecho, más $95 \%$ consideran que la construcción es un trabajo sólo para hombres, aludiendo a motivos de fuerza física. Mientras que el $98 \%$ coincide en que es un trabajo más adecuado para los hombres.

Esta opinión, y un concepto de la masculinidad basado en la fuerza y la demostración del valor pueden incitar, aun inconscientemente, a asumir ciertos riesgos, y ser un motivo más en algunos accidentes de trabajo. Aunque esta mentalidad parece que va desapareciendo poco a poco.

En este sentido, la mayoría defiende que las medidas de seguridad empleadas son totalmente necesarias, sólo un $25 \%$ de los entrevistados piensa que algunas son exageradas.

No obstante, en las entrevistas en contextos más informales, sobre todo entre los trabajadores, todavía era bastante común la idea de que las mujeres son, por lo general, más cuidadosas y precavidas que los hombres. Y esta vinculación de lo considerado femenino a la prevención y a al cuidado es un elemento que, de manera sutil, sigue teniendo relativa importancia para los riesgos laborales.

Si bien es cierto que, ante peligros muy evidentes o inmediatos, los trabajadores están más concienciados, en relación a los riesgos que desconocen o ven menos claros, los comportamientos se ven más proclives a ser influenciados por estos aspectos.

\section{Azar vs. prevención. Evitabilidad e inevitabilidad en los accidentes laborales}

Otra cuestión que consideramos interesante estudiar por la incidencia de los comentarios de la mayoría de los trabajadores es el de la suerte9 . Queríamos saber el lugar que ocupa la mala suerte para los trabajadores en los accidentes laborales y hasta qué punto son evitables para ellos con el uso de medidas de seguridad.

9 La idea de suerte estaría, en muchos casos, relacionada con la religión o religiosidad que se profese y en la creencia en el destino. Igualmente el uso de medallas, de figuras de santos, ciertos tatuajes, etc., a modo de amuletos, son indicadores de la presencia de las ideas de azar y destino y sus vínculos con las religiones. 
En líneas generales suelen afirmar que los accidentes son evitables (más del $75 \%$ ), y que cuando suceden por no usar las medidas de protección requeridas, es el propio accidentado el responsable de que haya sucedido y no se haya evitado.

Sin embargo, los datos cambian con formulaciones distintas de las preguntas, el $60 \%$ asocia el riesgo en el trabajo a la palabra accidente, más del 12\% a la probabilidad, frente al $26 \%$ que habla de una mala práctica en el trabajo.

Esto, consideramos que se debe a que la idea de la mala suerte (sólo el 10\% alude al azar como causa) y la influencia que pudiere tener en los accidentes laborales se haya en niveles más subconscientes, pues pensamos que los sujetos actúan condicionados por la cultura, y que esta conlleva aspectos más explícitos y otros más sutiles. Por ello, el análisis de estos últimos sólo puede llevarse a cabo con técnicas específicamente cualitativas. Es en la observación en el día a día, en los diálogos informales o mirando entre líneas en las entrevistas más formales cuando los elementos relacionados con el azar afloran.

En la rememoración y descripción de accidentes pasados por parte de los informantes hemos encontrado expresiones como "iqué mala suerte!”, "el pobre hombre...", "tuvo mala suerte y le sucedió..." o "tuvo la mala suerte de..."

Por ejemplo, un relato al que hacen alusión algunos trabajadores es el de un compañero que, caminando por un tejado, cayó por una claraboya que estaba en mal estado y se rompió. El hombre salvó su vida pero quedó con una discapacidad. El énfasis en la mala suerte que tuvo, en que precisamente se rompió el tejado cuando él estaba o en que no tenía por qué haberse roto en circunstancias normales, eclipsan datos como que el trabajador no llevaba puestos los arneses correspondientes que podían haber evitado los daños a su persona.

Como vemos, la creencia en el azar es un factor más que interfiere, junto a otros aspectos, en la percepción-construcción del riesgo que hacen los trabajadores. La poca probabilidad percibida de sufrir un accidente, el pensar que a uno no le va a pasar, que tan sólo es un momento para realizar un pequeño y sencillo trabajo, que va a estar más tiempo colocándose los EPI's que haciendo la tarea, que normalmente no le pasa nada a nadie, que sería mucha mala suerte que le sucediera a él, es un factor que debe ser tenido en cuenta.

Al respecto, Douglas (1996, pp. 57-58) nos habla del concepto de inmunidad subjetiva, un fuerte e injustificado sentido de falsa confianza que tienen los sujetos a la hora de actuar. Se tiende a minimizar la probabilidad de malos resultados en actividades muy familiares, subestimando los riesgos que se consideran controlados, ignorando los peligros cotidianos más comunes, pero también los de aquellos 
sucesos infrecuentes, de baja probabilidad. De esta forma el mundo inmediato parece más seguro, del mismo modo que se reducen los peligros distantes.

Otro ejemplo de ello se daba cuando planteábamos la situación ficticia de un compañero que se pone el casco a diario, pero que un día de mucho calor decide no usarlo y le cae un ladrillo en la cabeza. Casi todos los entrevistados elegían la opción de que había tenido mala suerte, por encima de que fuera culpa suya. Esto nos lleva además a la idea de que en ciertas circunstancias, en las que el uso de las medidas de protección se puede hacer incómodo, está aceptado en la mentalidad colectiva de los trabajadores que se prescinda de ellas.

\section{Incomodidad/comodidad}

En efecto, la incomodidad que suponen algunos EPI's para la relación correcta de ciertas tareas, supone una excusa para que se dejen de usar, como hemos podido constatar en nuestra investigación, y parece ser una factor recurrente que han encontrado otros autores (Espluga, 2004).

Así, nos hablan de que el casco les da calor en verano, de que los guantes le impiden la correcta movilidad manual, etc. El problema y la solución, podrían pasar por dos aspectos:

El primero está relacionado con una cuestión que radica en la que podríamos denominar "endoculturación laboral técnica". Como ya afirmara Mauss (1991), en la misma naturaleza de la técnica se encuentra la eficacia y, por ello, en cualquier cultura, se intenta economizar la motricidad en función de la misma. En los procesos de aprendizaje técnico encontramos momentos de experimentación que nos pueden llevar a buscar los caminos más cortos para la realización de una tarea. Es por ello, que en estos procesos, debería inculcarse como imprescindible el uso de ciertos EPI's, y no dejarlas a la elección del aprendiz. Una vez se han adquirido unas habilidades técnicas y se ha encontrado la mayor eficacia, se crean habitus (Bourdieu, 1997) incorporados, que resultan difíciles de modificar o de entorpecer sus acciones con elementos externos.

El segundo, siguiendo las ideas de algunos informantes, se halla en la mala ergonomía de estos utensilios. Pero este es otro problema técnico que siempre está en proceso de mejora. Si bien se encuentra en manos de las empresas el adquirir y proporcionar a sus trabajadores el material mejor evolucionado tecnológicamente para este fin. 


\section{Impacto de los accidentes sobre la percepción y actuación de los trabajadores}

La mayoría de los trabajadores con un mínimo de experiencia ha vivenciado algún tipo de accidente en el trabajo. Casi todos conocen a alguien que ha sufrido algún percance de relativa gravedad. Sólo los trabajadores más nóveles se han librado de ellos.

En términos generales un $61 \%$ afirman tener compañeros que han tenido algún accidente de trabajo, mientras que un 32\% lo han sufrido ellos mismos.

La vivencia de una situación de esta índole, siempre en función de la gravedad, suele representar un suceso de especial relevancia en la vida laboral de los trabajadores. Las expresiones en sus rostros, su tono de voz, $\mathrm{u}$ otros aspectos gestuales cuando les pedimos que nos cuenten cómo fue el suceso, sugieren que estos acontecimientos han sido más o menos traumáticos para nuestros informantes. Generalmente pasan a formar parte de la memoria colectiva de los trabajadores y difícilmente se olvidan.

Por ello, el $91 \%$ de los trabajadores dicen hacer más uso de las medidas de protección desde que se produjo el accidente, y el $87 \%$ que la empresa ha tomado más medidas desde entonces.

Tal y como nos han contado nuestros informantes, podríamos generalizar que la influencia de los accidentes en los hábitos laborales, entre los que nos interesan las intenciones preventivas, está condicionada por dos factores.

Por un lado, la gravedad percibida del accidente. Lo que resulta evidente es que cualquier accidente que deje secuelas irreversibles sobre el cuerpo del trabajador, a modo de discapacidad, o temporales que impliquen hospitalización son percibidas como graves. Éstas últimas sobre todo cuando la vida haya corrido peligro.

Por otro lado, el tiempo es un factor que aleja o acerca la presencia del accidente en los discursos y prácticas de la cotidianeidad laboral. De modo que, con el tiempo, y en función de la gravedad, los accidentes tienden, si no al olvidarse, sí a desaparecer de la cotidianidad en el ámbito de trabajo. Y así, poco a poco vuelven a incidir y adquirir relevancia de nuevo factores como la probabilidad y el azar, la incomodidad, etc., que empujan hacia la relajación en las costumbres que implican la prevención de los riesgos laborales y la "ausencia" del miedo.

Algunas empresas han adoptado estrategias, más o menos conscientemente, que se encargan de recordar de manera constante las posibles consecuencias, nefastas para la salud, de una relajación en uso de las medidas de prevención. Tal es el ejemplo de una empresa que encargó a un trabajador aquejado de una 
invalidez producida en el ámbito laboral, la supervisión y tutorización de los trabajadores en cuestiones preventivas.

\section{Reflexiones finales}

Hemos visto cómo hay una serie de factores culturales que tienen repercusiones directas en la administración del riesgo por parte de empresarios y empleados de la construcción.

Pero, junto a los factores que hemos ido explicando, existen otros muchos que condicionan tanto la percepción y construcción que se hace del riesgo, como la disposición a adoptar las medidas de prevención necesarias.

Uno de ellos es el elemento de obligación, relacionándolo con las repercusiones a nivel de una posible pérdida del puesto de trabajo que puede suponer su incumplimiento de las normativas.

Ya hemos comentado como los empresarios técnicos de algunas empresas nos contaban cómo, a veces, tienen ciertas dificultades para que sus trabajadores usen los EPI's, quienes aluden especialmente a factores como la incomodidad, la economización del tiempo, etc. Para ellos los trabajadores cumplen con las medidas de prevención por obligación, por el continuo control por parte de la empresa y las consecuencias negativas en su puesto laboral que podría acarrear la desobediencia de las normativas.

En efecto, algunos trabajadores reconocían que acataban el uso de las protecciones para evitar "la bronca del jefe", por encima de otros motivos.

En estos aspectos se produce una tensión dentro de las mismas empresas de valores y actuaciones que en principio pueden parecer contradictorios. De un lado, se intenta mantener el ritmo laboral más alto posible para incrementar los beneficios, donde muchas medidas de prevención entorpecen y retrasan el trabajo. Del otro, los accidentes laborales y el incumplimiento de normativas pueden suponer para la empresa considerables sanciones económicas. Con ambas motivaciones contrapuestas deciden sus procedimientos de actuación que se llevan a la práctica en el día a día y el grado de supervisión y disciplina que se impone a los trabajadores con respecto a las medidas de prevención. A estos dos también se le añade el valor que se da a la salud de los propios trabajadores.

Por parte de los trabajadores parece claro que la formación, la información, la salud, el azar, la incomodidad y la masculinidad son otros aspectos que inciden en sus comportamientos. 
Por último, resulta significativo que casi ningún informante situaba el riesgo como factor prioritario a la hora de decidir abandonar u optar por un trabajo. Ubicándose muy por encima otros como la remuneración, los horarios o el esfuerzo físico requerido por el empleo.

El valor que se da al dinero y la riqueza, al tiempo y su ahorro o al esfuerzo físico como más duro que el intelectual, son también ideas culturales que condicionan las actuaciones de los sujetos, y que complejizan todavía más, la labor de la prevención de riesgos laborales. En ellos profundizaremos en futuras investigaciones.

Por todo esto se hace necesario ubicar a los trabajadores en sus contextos de trabajo, a éstos en la sociedad y en la cultura, y adoptar una perspectiva holística, para comprender mejor los procesos que se dan en la construcción del riesgo laboral y su prevención.

\section{Bibliografía}

Beck, Ulrich (1986). La sociedad del riesgo. Hacia una nueva modernidad. Barcelona: Paidós.

Bestard, Joan (1996). Prólogo. En: Mary Douglas, La aceptabilidad del riesgo según las ciencias sociales. Barcelona: Paidós.

BOE (Boletín Oficial del Estado) (2012). V Convenio colectivo del sector de la construcción. $\mathrm{N}^{\mathrm{o}}$ 64, jueves 15 de marzo de 2012.

(2015). Prevención de riesgos laborales. Madrid: Agencia Estatal Boletín Oficial del Estado.

Borgia et al. (1997). Valutazione dei corsi per l'abilitazione all'aquisto ed uso dei fitofarmici. La Medicina del Lavoro 5(88), 416-424.

Bourdieu, Pierre (1997) Razones prácticas. Sobre la teoría de la acción. Barcelona: Anagrama.

Cobos, David, Pérez, Itahisa y Reyes, Encarnación (2011). La visión sobre los riesgos laborales y la cultura preventiva de los estudiantes universitarios: Un estudio exploratorio en la universidad Pablo Olavide. Bordón. Revista de pedagogía, 63(3), 75-90.

Connell, Robert W. (1995). Masculinities. Berkeley: University of California Press.

Douglas, Mary (1996). La aceptabilidad del riesgo según las ciencias sociales. Barcelona: Paidós.

Douglas, Mary y Wildavsky, Aaron (1982). Risk and Culture. An Essay on the Selection of Technical and Environmental Dangers. Berkeley: University of California Press. 
Espluga, Josep (2004). La exposición laboral a pesticidas en la agricultura. Un caso aparente de subestimación del riesgo. Sociología del Trabajo, 51, 33-60.

Fischhoff, B. et al. (1978). How Safe is Safe Enough, A Psychometric Study of Attitudes towards Technological Risk and Benefits. Policy Sciences, 8, 127-152.

Frías, Antonio (2006). La cultura y las conductas de riesgo en adolescentes (Tesis de Doctorado). Granada: Universidad de Granada.

Jociles, María Isabel (2001). El estudio de las masculinidades. Panorámica general. Gazeta de Antropología, 17, 1-14.

Martínez Guirao, Javier Eloy (2004). Los gimnasios ‘deportivos'. Del ocio a la obligación. En: Antón Álvarez Sousa (Coord.), Turismo, ocio y deporte (pp. 131140). La Coruña: Universidad de La Coruña,

Mauss, Marcel (1991). Sociología y antropología. Madrid: Tecnos.

Minello Martini, Nelson (2002). Masculinidad/es. Un concepto en construcción. Nueva antropología, XVIII (61), 11-30.

Montenegro, Silvia M. (2005). La sociología de la sociedad del riesgo: Ulrich Beck y sus críticos. Pampa. Revista Interuniversitaria de Estudios Territoriales, 1(1), 117-130.

Moreno, Isidoro (1991). Identidades y rituales. Estudio introductorio. En: Joan Prat et al. (Eds.), Antropología de los pueblos de España (pp. 601-636). Madrid: Taurus.

Reig, Adela y Clemente, Miguel (2010). Análisis de la Ley de Prevención de Riesgos Laborales, desde los puntos de vista de la prevención y de la Psicología de las Organizaciones. Anuario da Facultade de Dereito da Universidade da Coruña, 14, 265-276.

Slovic, P., Fichtenstein, S. y Fischhoff, B. (1979). Images of Disaster: Perception and Acceptance of Risk from Nuclear Power. En: G. Goodman y W. Rowen (Eds.), Energy Risk Manegement. Londres: Academic Press.

Téllez, Anastasia (2002). Trabajo, identidad y género: la puesta en juego de las representaciones ideológicas. Cuadernos de Relaciones Laborales, 20(1), 191-214. (2013). El análisis de la adolescencia desde la antropología y la perspectiva de género. Rev. Interacções, 25, 52-73

Urteaga, Eguzki (2012). Los determinantes culturales de la percepción social del riesgo cultural. Argumentos de Razón Técnica, 15, 39-53.

Zonabend, François (1989). La presqu'île au nucléaire. Paris: Odile Jacob.

Fecha de recepción: 25/7/2015; fecha de aprobación: 30/11/2015 